* Possui mestrado em Análise Econômica do Direito pela Universidade Católica de Brasília (2014), bacharelado em Direito pela Universidade Federal de Ouro Preto (2008) e 17 artigos publicados em periódicos classificados pelo sistema Web Qualis - CAPES. Além de consultor, atualmente é professor do Centro Universitário de Brasília (Uni CEUB), advogado associado da Pinheiro \& Mello Advogados $(\mathrm{P} \& \mathrm{M})$ e pesquisador do Grupo de Pesquisa de Inovação em Saúde (GIS). Tem expe-riência na área do Direito, com ênfase em Direito Empresarial, Propriedade Intelectual e Análise Econômica do Direito. Email: rafael.dany@gmail. com

**Advogada inscrita no quadro da OAB-DF. Mestre em Desenvolvimento e Políticas Públicas (Saú-de Pública) pela Fundação Oswaldo Cruz (Fiocruz). E-mail: tatiana. nogueira@saude.gov.br

*** Doutor em Economia pela Universidade Católica de Brasília (UCB), Mestre em Desenvolvimento Local (UCDB) e Administrador. Professor em 2 Programas de Pós-graduação da UCDB: Sustentabilidade Agropecuária, em nível de Mestrado e Doutorado e no Mestrado em Desenvolvimento Local. Pesquisador da área de Economia, Estatística (Métodos Quantitativos) e Economia Agrícola com ênfase em Microeconomia Aplicada com Econometria. Pesquisador Visitante do Instituto de Pesquisa Econômica e Aplicada - IPEA-Brasília/DF. Pesquisador do CeTeAgro - Centro Tecnológico do Agronegócio da Universidade Católica Dom Bosco - Campo Grande - MS. Editor associado da Economic Analysis of Law Review. E-mail: michelangelo 111@gmail.com

\section{O Estudo Trilateral (OMPI, OMC e OMS) e a Promoção do Acesso à Saúde}

\section{The Trilateral Study (WIPO, WTO and WHO) and the Promotion of Health Care ACCESS}

\author{
* Dany Rafael Fonseca Mendes \\ ** Tatiana Siqueira Nogueira \\ *** Michel Angelo Constantino de Oliveira
}

Resumo: Este trabalho possui como objetivo a análise do estudo trilateral elaborado pela Organização Mundial da Propriedade Intelectual, Organização Mundial do Comércio e Organização Mundial da Saúde, relacionando a propriedade intelectual, o comércio internacional e a saúde pública, cujo escopo visa à promoção do acesso a novas tecnologias médicas, principalmente pelas populações mais pobres, para que, com isso, a população global alcance o mais alto nível de saúde possível. O estudo em referência é uma resposta a uma demanda crescente, especialmente em relação aos países de baixo desenvolvimento relativo, por desenvolvimento, por fortalecimento de suas capacidades para definir políticas nas áreas de intersecção entre saúde, comércio e propriedade intelectual, possibilitando, com isso, acesso e inovação de medicamentos e de outras tecnologias médicas. Para tanto, nesta pesquisa, foi elaborado um estudo a respeito das três organizações, suas origens, funcionamentos e principais atribuições. Além disso, o presente artigo aborda acerca de outros aspectos contidos no estudo trilateral, como os papéis de cada uma das organizações, a carga global de doenças, o desafio essencial para a política de saúde, a relação entre inovação e dimensões de acesso, além de outros temas abordados pelo estudo trilateral.

Palavras-chave: Estudo trilateral. Propriedade intectual. Comércio internacional. Saúde pública.

Abstract: This work aims to analyze the trilateral study by the World Intellectual Property Organization, World Trade Organization and World Health Organization, relating to intellectual property, international trade and public health, whose scope is aimed at promoting access to new medical technologies, especially the poorest, so that with this, the global population will reach the highest possible level of health. The study in reference is a response to a growing demand, especially 
in relation to low relative development countries for development by strengthening their abilities to define policies in the areas of intersection between health, trade and intellectual property, allowing, thus, access and innovation of drugs and other medical technologies. To this end, in this research, we designed a study about the three organizations, its origins, workings and main duties. Additionally, this article discusses about other aspects contained in the trilateral study, as the roles of each organization, the global burden of disease, the key challenge for health policy, the relationship between innovation and dimensions of access, in addition other themes addressed by the trilateral study.

Keywords: Trilateral study. Intelectual property. International trade. Public health. 


\section{INTRODUÇÃO}

O estudo elaborado em conjunto pelas Agências da Organização das Nações Unidas $\left(\mathrm{ONU}^{1}\right)$ é uma análise dos três temas envolvidos - propriedade intelectual (PI), comércio internacional e saúde pública - para promover o acesso, especialmente das populações mais pobres, a novas tecnologias médicas no âmbito de acordos relacionados aos objetos de análise da publicação. $\mathrm{O}$ documento observa que, em uma era da globalização, os progressos realizados na saúde pública de qualquer país têm um impacto positivo sobre a comunidade internacional como um todo, e, consequentemente, a cooperação internacional em saúde pública é essencial para o desenvolvimento sustentável do planeta (WORLD HEALTH ORGANIZATION, 2012). Afinal, com a redução das distâncias físicas, provocadas pela evolução dos meios de transporte, e das de interação, provocadas pelos saltos de tecnologia da informação, não é mais possível manter um país plenamente saudável sem considerar a saúde dos demais membros da população mundial.

$\mathrm{Na}$ apresentação das propostas do documento (WORLD HEALTH ORGANIZATION, 2012), tem-se que o objetivo fundador da Organização Mundial da Saúde é que todos os povos alcancem o mais alto nível possível de saúde. Vinculadas a essa meta, a Organização Mundial da Propriedade Intelectual e a Organização Mundial do Comércio têm intensificado o trabalho em suas respectivas áreas de atuação para apoiar os esforços globais para a melhoria dos resultados mundiais em saúde. Cientes de que não é possível separar inovação de acesso, o foco do estudo foi ampliado para considerar: os requisitos de proteção da propriedade indispensáveis para promover a inovação; e o atendimento às necessidades básicas de saúde negligenciadas, garantindo o acesso equitativo a todas as tecnologias médicas vitais.

Portanto, ao contrário de propostas menos arrazoadas de algumas organizações que lutam pelo direito à saúde, não é possível dissociar o acesso à saúde da inovação em saúde, tampouco é possível promover o acesso à saúde em desrespeito a acordos comerciais e de propriedade intelectual.

\footnotetext{
"The United Nations is an international organization founded in 1945. It is currently made up of 193 Member States. The mission and work of the United Nations are guided by the purposes and principles contained in its founding Charter. Due to the powers vested in its Charter and its unique international character, the United Nations can take action on the issues confronting humanity in the 21 st century, such as peace and security, climate change, sustainable development, human rights, disarmament, terrorism, humanitarian and health emergencies, gender equality, governance, food production, and more" (UNITED NATIONS, 2015a).
} 
No sentido de continuar promovendo a inovação tecnológica, majorando o acesso, o estudo esclarece que as três Agências têm razões de sobra para, numa ação coordenada, cooperar mais estreitamente em questões como: padrões de inovação e acesso; fatores legais e políticos que afetam a produção; e difusão de tecnologias médicas (WORLD HEALTH ORGANIZATION, 2012). Futuramente, esses fatores legais e políticos analisados pelo estudo trilateral podem servir de base para a formulação de políticas públicas mais assertivas.

A declaração sobre TRIPS² e Saúde Pública (Declaração de Doha ${ }^{3}$ ) já trazia entre seus embasamentos a produção de ideias sobre essas interseções (entre a saúde pública, o sistema de propriedade intelectual, regras de comércio e concorrência), além de medidas para promover a inovação e o acesso a tecnologias médicas. O estudo é, portanto, um estreitamento da cooperação trilateral das Agências guiado pela abordagem em matéria de saúde pública que, por sua vez, tem sido catalisada pela Declaração de Doha, pela Agenda de Desenvolvimento ${ }^{4}$ da OMPI, pela Estratégia Global ${ }^{5}$ da OMS e pelo Plano de Ação sobre Saúde Pública, Inovação e Propriedade Intelectual ${ }^{6}$, também desta Organização. Além do estreitamento colaborativo entre as Organizações,

${ }^{2}$ O Acordo sobre os Aspectos dos Direitos de Propriedade Intelectual, ou TRIPS (do inglês Agreement on Trade-Related Aspects of Intellectual Property Rights), é um tratado multilateral, integrante do conjunto de acordos assinados em 1994, durante a criação da Organização Mundial do Comércio.

3 Em Novembro de 2001, durante a IV Conferência Ministerial da OMC, foi assinada no Qatar a Declaração de Doha, nas quais uma gama de matérias é negociada, em temas como agricultura, serviços, acesso de produtos não agrícolas ao mercado, dentre outros. Nessa Declaração, também são discutidas e negociadas a relação entre a saúde pública e os direitos de propriedade intelectual (implementando e interpretando o Acordo TRIPS de forma a apoiar a saúde pública), por meio tanto do acesso aos medicamentos existentes, quanto à criação de novos fármacos (WORLD TRADE ORGANIZATION, 2015).

${ }^{4}$ A Agenda de Desenvolvimento da OMPI, estabelecida em 2007, adotou 45 recomendações, agrupadas em seis grupos, e foi um passo importante na organização, visto que assegurou que as considerações sobre o desenvolvimento sejam parte integral dos trabalhos da OMPI (WORLD INTELLECTUAL PROPERTY ORGANIZATION, 2015).

5 A estratégia global da OMS sobre saúde pública, inovação e propriedade intelectual propõe que a organização deveria ter uma posição estratégica e central no relacionamento entre saúde pública e os dois outros componentes, por meio da promoção da inovação e do acesso a medicamentos, o que, ao invés de motivar as pesquisas lastreadas apenas no mercado, incentivaria a pesquisa relacionada ao combate às doenças que afetam a população em países em desenvolvimento (WORLD HEALTH ORGANIZATION, 2011).

${ }^{6}$ O plano de ação, que está relacionado à Estratégia Global sobre saúde pública, inovação e propriedade intelectual, possui 108 ações específicas que, juntamente com os oito elementos da estratégia, buscam, dentre outras coisas: estabelecer, priorizar e promover a pesquisa; adotar a construir capacidade de inovação; promover a transferência de tecnologia e a produção local de produtos médicos; promover o gerenciamento e aplicação dos direitos de propriedade intelectual visando à melhoria da saúde pública; promover o acesso aos produtos médicos (WORLD HEALTH ORGANIZATION, 2011). 
o objetivo fundamental do estudo trilateral é fornecer uma plataforma sólida para o debate político futuro e para a formulação de outras análises sobre o tema (WORLD HEALTH ORGANIZATION, 2012). No âmbito da formulação de políticas públicas para o Brasil, a Secretaria de Ciência, Tecnologia e Insumos Estratégicos do Ministério da Saúde (SCTIE/MS ${ }^{7}$ ) é a representação do governo com mais motivos para manter a atenção no equilíbrio entre incentivo à inovação e restrição de acesso. Afinal, se, por um lado, uma das atribuições da SCTIE/MS é garantir o desenvolvimento do Complexo Econômico-Industrial da Saúde $\left(\mathrm{CEIS}^{8}\right)$, reduzindo a vulnerabilidade tecnológica do País perante fornecedores internacionais (MDIC), por outro, sendo uma das peças-chave do Ministério da Saúde, a mesma Secretaria tem o objetivo fundamental de garantir acesso à saúde pública para a população brasileira.

Inicialmente este trabalho buscou apresentar uma introdução lastreada no prefácio elaborado pelos próprios diretores das três Agências, além de descrever, ainda alicerçado naquele preâmbulo, os objetivos norteadores do estudo trilateral em si. Antes de retornar à análise do estudo realizado pelas Organizações, o trabalho aqui exposto apresentará uma descrição sucinta de cada Agência, exibindo, mais adiante, um resumo das atividades e objetivos da Organização Mundial da Propriedade Intelectual, da Organização Mundial do Comércio e da Organização Mundial da Saúde. De volta ao exame do estudo trilateral, o presente trabalho exporá um resumo de cada capítulo daquele texto e, concluindo, fará uma análise crítica das propostas elaboradas pelas Agências da ONU, indicando, se possível, uma avaliação do posicionamento mais adequado ao Estado brasileiro na promoção da saúde e na manutenção do delicado equilíbrio entre inovação e acesso.

\footnotetext{
${ }^{7}$ A Secretaria de Ciência, Tecnologia e Insumos Estratégicos do Ministério da Saúde possui como objetivo fortalecer o SUS como sistema de saúde universal, por meio do desenvolvimento da capacidade científica, tecnológica e produtiva nacional. Nesse sentido, a SCTIE/MS tanto formula, quanto implementa, políticas nacionais voltadas à ciência, tecnologia e inovação em saúde, assistência farmacêutica e fomento à pesquisa, desenvolvimento e inovação na área de saúde (BRASIL, 2013).

8 "Apesar de os segmentos que compõem o CEIS possuírem dinâmicas bastante diversas entre si, eles compartilham o mesmo arcabouço político institucional, ou seja, o ambiente regulatório, as diretrizes de política social, econômica, industrial e a estrutura político-institucional do sistema nacional de saúde. Assim, o mesmo ambiente político institucional comporta uma gama variada de atores com objetivos diversos e muitas vezes conflitantes, evidenciando a necessidade da atuação do Estado como mediador dos interesses sanitários e daqueles mais característicos de mercado, envolvidos na saúde. Em decorrência dessa complexidade faz-se mister uma visão integrada desse conjunto interligado de produção de bens e serviços em saúde, entendendo-os como atividades produtivas interdependentes, o que remete ao arcabouço teórico da economia política adotado nesta análise" (GADELHA; COSTA; MALDONADO, 2012).
} 


\section{AGÊNCIAS}

\subsection{Organização Mundial da Propriedade Intelectual}

A OMPI é uma entidade internacional de Direito Internacional Público com sede em Genebra, Suíça, integrante do Sistema das Nações Unidas que atualmente é composta de 185 Estados-membros e administra 24 tratados internacionais. Criada em 1967, é uma das 16 agências especializadas da ONU e tem por propósito a promoção da proteção da propriedade intelectual ao redor do mundo, através da cooperação entre Estados, além do uso da propriedade intelectual como meio de promoção da inovação e criatividade (WORLD INTELLECTUAL PROPERTY ORGANIZATION, 2013).

Segundo informações disponibilizadas pela Organização Mundial da Propriedade Intelectual (WORLD INTELLECTUAL PROPERTY ORGANIZATION, 2013), a Agência promove o desenvolvimento e uso do sistema internacional de propriedade intelectual, principal e funcionalmente, por meio das seguintes ações: executando sistemas que tornam mais fácil a obtenção e proteção internacional de patentes, marcas, desenhos e denominações de origem; resolvendo disputas de propriedade intelectual; ajudando no desenvolvimento do quadro legal internacional de PI em conformidade com as necessidades de evolução da sociedade; erguendo redes de colaboração e plataformas técnicas para compartilhar conhecimentos e simplificar as operações de PI, incluindo bases de dados gratuitas e ferramentas para a troca de informações; e construindo capacitação no uso de propriedade intelectual para apoiar o desenvolvimento econômico.

Em resumo, a OMPI trabalha com seus Estados-membros e as partes interessadas para melhorar a compreensão e respeito por PI no mundo todo, fornecendo análises econômicas e estatísticas, e contribuindo, com soluções baseadas em propriedade intelectual, para o enfrentamento de desafios globais (WORLD INTELLECTUAL PROPERTY ORGANIZATION, 2013).

\subsection{Organização Mundial do Comércio}

A OMC é uma organização internacional que trata das regras do comércio internacional e, atualmente, conta com 156 Estados-membros. O surgimento da $\mathrm{OMC}$ foi um importante marco na ordem internacional que começara a ser delineada no fim da Segunda Guerra Mundial. A Agência surgiu a partir dos preceitos estabelecidos pela Organização Internacional do Comércio (OIC), consolidados na Carta de Havana, e, uma vez que esta não foi levada adiante 
pela falta de aceitação do Congresso dos Estados Unidos da América (WORLD TRADE ORGANIZATION, 2013). Oficialmente, a OMC passou a valer como agência da ONU a partir de $1^{\circ}$ de janeiro de 1995, e todas as suas realizações são o resultado das negociações das quais é foro. A maior parte do trabalho atual da OMC vem das negociações ocorridas entre 1986 e 1994, chamadas de Rodada Uruguai, e daquelas ocorridas no âmbito do Acordo Geral sobre Tarifas e Comércio - GATT, sigla em inglês (WORLD TRADE ORGANIZATION, 2013).

Sendo gerida por seus Estados-membros, a Organização Mundial do Comércio tem como básicas as seguintes funções (WORLD TRADE ORGANIZATION, 2013): os acordos da OMC abrangem bens, serviços e propriedade intelectual e incluem compromissos de cada país com tarifas aduaneiras mais baixas e com a redução de outras barreiras comerciais, para abrir mercados e para mantê-los abertos (além disso, os acordos estabelecem procedimentos para a resolução de litígios e, não sendo estáticos, podendo ser renegociados de tempos em tempos); vários conselhos e comitês da OMC buscam garantir que esses requisitos estejam sendo seguidos e que os acordos da Organização estejam sendo devidamente implementados; o sistema de solução de controvérsias é um procedimento para resolver disputas comerciais no âmbito de atuação da Organização, as quais são julgadas por peritos independentes e são baseadas em interpretações dos acordos e compromissos de cada país; seja através de prazos maiores para a implementação de acordos, seja pela disponibilização de cursos de capacitação, uma das funções da OMC é ajudar os países em desenvolvimento a ampliar sua infraestrutura necessária à expansão do comércio, construindo capacidades comerciais; e, mantendo um diálogo regular com organizações não-governamentais, parlamentares, outras organizações internacionais, mídia e com o público em geral sobre os vários aspectos das negociações em curso, a OMC pretende reforçar a cooperação e aumentar a consciência sobre suas atividades.

\subsection{Organização Mundial da Saúde}

Com sede em Genebra, a OMS é uma agência especializada em saúde, fundada em 1948 e, como as demais, subordinada à Organização das Nações Unidas. Após as guerras do fim do século XIX e, principalmente, com o fim da Primeira Guerra Mundial, a SDN (Sociedade das Nações), também conhecida como Liga das Nações, precursora da ONU, criou seu comitê de higiene, o qual foi o embrião da Organização Mundial da Saúde. A OMS é a agência 
responsável por prover liderança em questões de saúde globais, definição da agenda de pesquisa em saúde, estabelecimento de normas e padrões, articulando opções de políticas de saúde baseadas em evidências, fornecendo apoio técnico aos países e acompanhando e as tendências de saúde. Contando com atuais 193 Estados-membros, a OMS considera que, dadas as necessidades do século XXI, a saúde é uma responsabilidade partilhada, envolvendo o acesso equitativo a cuidados essenciais e a de defesa coletiva contra as ameaças transnacionais (WORLD HEALTH ORGANIZATION, 2013).

Com a visão supramencionada e o foco em saúde pública, a Organização tem como funções essenciais (WORLD HEALTH ORGANIZATION, 2013): prover a liderança em questões críticas para a saúde e o envolvimento em parcerias onde a ação comum seja fundamental; determinar a agenda de pesquisa e estimular a geração, difusão e utilização de conhecimentos valiosos; estabelecer normas e padrões, além de acompanhar a aplicação prática dessas normas e padrões; desenvolver opções de políticas lastreadas na ética e nas ciências; prestar apoio técnico, catalisando mudanças e capacitação institucional sustentável; e acompanhar a situação de saúde e avaliar suas tendências.

\section{OESTUDO TRILATERAL}

Como mencionado no item 1 , o estudo sobre o qual se baseia o presente trabalho surgiu a partir de um programa permanente de cooperação entre as agências da ONU especializadas em propriedade intelectual (item 2.1), comércio (item 2.2) e saúde (item 2.3). Também de acordo com a "Introdução", o estudo trilateral responde a uma demanda crescente, particularmente dos países de baixo desenvolvimento relativo ${ }^{9}$ em desenvolvimento, de robustecimento da capacidade para a definição de políticas nas áreas de intersecção entre saúde, comércio e propriedade intelectual, com foco no acesso e na inovação de medicamentos e de outras tecnologias médicas.

A necessidade de cooperação e de coerência no plano internacional mencionada acima tem sido intensificada ao longo da última década, como demonstram as sucessivas decisões multilaterais o corridas desde o início dos

\footnotetext{
9 "Os países de menor desenvolvimento relativo (least-developed countries - LDCs) são aqueles, designados pela ONU, que possuem as mais baixas receitas, medidas pelo Produto Interno Bruto dos países, recursos humanos escassos e um baixo nível de diversificação econômica. Nesses países, o acesso à alimentação, saúde, educação, habitação e infraestrutura é precário, sendo que ainda há grandes deficiências quanto à distribuição de renda" (INSTITUTO DE ESTUDOS DO COMÉRCIO E NEGOCIAÇÕES INTERNACIONAIS, 2015).
} 
anos 2000, quais sejam: United Nations General Comment on the Right to Health (2000); WTO Doha Declaration on the TRIPS Agreement and Public Health (2001); WHO-WTO joint study WTO Agreements and Public Health (2002); WTO creates new TRIPS flexibility for access to medicines in countries lacking manufacturing capacity (2003/2005); WHO Commission report on Public health, innovation and intellectual property rights (2006); WIPO Development Agenda (2007); WHO Global Strategy and Plan of Action on Public Health, Innovation and Intellectual Property (2008); WHO-WIPO-WTO trilateral cooperation commences (2009). O estudo trilateral está estruturado de forma a permitir que seus usuários entendam os fundamentos da política, para, em seguida, investigar mais profundamente áreas de interesse particular. Trata-se, portanto, de uma fonte para construção de capacidades destinada a tomadores de decisões políticas (WORLD HEALTH ORGANIZATION, 2012).

O estudo elaborado pelas referidas Agências apresenta o contexto geral da política de saúde relativa às tecnologias médicas, define os papéis distintos e mandatos das três Agências de cooperação, e descreve a carga global de doenças que delineia o desafio essencial para a política de saúde. Contra o pano de fundo da carga global de doenças e riscos para a saúde global, o estudo apresenta o imperativo fundamental para a colaboração entre os vários intervenientes interessados em tecnologias médicas. Ele também demonstra a necessidade de uma abordagem coordenada, tendo em conta a saúde, a propriedade intelectual e as variáveis de comércio, de forma a garantir tomadas de decisão coerentes na área de saúde pública em nível internacional, regional e nacional.

A OMS, a OMPI e a OMC têm mandatos distintos, embora complementares, para trabalhar sobre questões relacionadas com a saúde pública, a propriedade intelectual e o comércio. Ainda que os principais desenvolvimentos internacionais digam respeito a medicamentos, o estudo trilateral abrange também outras tecnologias, tais como vacinas e dispositivos médicos, incluindo os de diagnóstico, devido à sua importância para a obtenção de resultados de saúde pública. Os tomadores de decisões políticas em matéria de PI e de saúde pública são confrontados com a difícil tarefa de identificar a combinação ideal de opções para melhor promover os seus objetivos nacionais. Os governos estão, portanto, sempre buscando informações mais coerentes, abrangentes e acessíveis para o debate político. Nesse sentido, o décimo aniversário da adoção da Declaração de Doha proporcionou uma excelente oportunidade para colher a experiência necessária para melhorar o acesso, 
promovendo a inovação médica. Num ambiente de cooperação, o estudo trilateral foi concebido para servir de mapa de referência para os tomadores de decisões políticas no sentido mais amplo - legisladores, funcionários do governo, representantes de organizações internacionais, organizações não-governamentais e pesquisadores.

Dentro do sistema das Nações Unidas, a OMS é a autoridade de direção e coordenação para a saúde, sendo é responsável por fornecer a liderança em questões de saúde global, por influenciar a agenda de pesquisa em saúde, estabelecer normas e padrões, articular opções políticas baseadas em evidências, fornecer apoio técnico aos países e, ainda, acompanhar e avaliar as tendências de saúde. A OMPI é a Agência especializada das Nações Unidas dedicada ao desenvolvimento de um sistema de propriedade intelectual equilibrado e acessível que premia a criatividade, que estimula a inovação e que, no interesse público, contribui para o desenvolvimento econômico. A missão principal da OMC é a abertura global do comércio, bem como desenvolver e manter um sistema de comércio internacional baseado em regras claras. Dado que a parceria é fundamental para uma resposta internacional eficaz aos desafios em constante evolução, as Secretarias da OMS, da OMPI e da OMC têm intensificado a colaboração entre agências em assuntos relacionados com a saúde pública, a propriedade intelectual e o comércio (WORLD HEALTH ORGANIZATION, 2012).

\subsection{Diagnóstico}

Compreender a evolução da carga global de doenças (GBD, sigla em inglês) e o papel dos principais riscos para a saúde é importante, a fim de desenvolver estratégias eficazes para melhorar a saúde global e também a fim de identificar a gama de tecnologias médicas necessárias ao enfrentamento do problema. Grandes declínios nas taxas de mortalidade entre 2004 e 2030 foram projetados para todas as principais causas transmissíveis, maternas, perinatais e nutricionais da morte, incluindo HIV, tuberculose e malária. O envelhecimento da população em países de baixa e média renda resultará em um aumento significativo no número total de mortes por doenças não transmissíveis ao longo dos próximos 25 anos - globalmente, estas doenças representarão mais de três quartos de todas as mortes projetadas para 2030. Os principais riscos globais para a mortalidade do mundo são a hipertensão arterial, o uso do tabaco, a glicemia elevada, o sedentarismo e a obesidade. Entre os principais riscos globais para a carga de doenças estão: as crianças abaixo do peso e o sexo inseguro, 
seguidos pelo uso de álcool e pelo consumo de água não potável, além da falta de saneamento e higiene. Para o enfrentamento dos desafios apresentados pela GBD, seria necessário aumentar a diversidade de tecnologias médicas, evoluindo dentro de um contexto mais amplo de medidas preventivas com foco no estilo de vida, nutrição e fatores ambientais (WORLD HEALTH ORGANIZATION, 2012).

Segundo as três Agências WHO, WIPO e WTO, a obtenção de resultados sustentáveis em saúde pública depende da interação dinâmica da política nacional de saúde, incluindo sistemas de promoção da saúde e de financiamento adequados. Tudo isso em um ambiente regulatório com configurações de comércio e concorrência, políticas de contratos, estratégias de inovação e de propriedade intelectual (WORLD HEALTH ORGANIZATION, 2012). Os processos políticos da última década levaram a uma melhor compreensão de como esses componentes políticos distintos podem e devem trabalhar juntos para a produção de resultados voltados à saúde pública, buscando sinergias positivas entre os direitos humanos, saúde, acesso, inovação e dimensões comerciais. Nesse ambiente de interação, a inovação não pode ocorrer de forma isolada e o acesso deve ser visto num contexto mais amplo, incluindo a necessidade de inovação e de uma regulamentação eficaz. Assim, a maior disponibilidade e amplitude de dados em cada um desses domínios da política oferece uma rica base empírica para a tomada de decisões.

O estudo trilateral delineia também os elementos essenciais do quadro internacional - das políticas de saúde, de propriedade intelectual e de comércio - para, em seguida, produzir uma análise mais detalhada da inovação e das dimensões de acesso. O texto descreve as ideias-chave da economia de inovação de tecnologias médicas e do acesso, tendo em vista a crescente utilização de conceitos econômicos para informar discussões de políticas de saúde. O estudo descreve ainda o cenário político, com foco em como os elementos se cruzam, com particular ênfase nas tecnologias médicas. Nesse contexto, o quadro político descrito compreende a política, os recursos econômicos e jurídicos dos sistemas de PI e inovação, a regulamentação de produtos médicos, a política de concorrência e as medidas de política comercial relevantes, incluindo as tarifas de importação, as regras sobre o comércio de serviços, as compras governamentais, além dos acordos de livre comércio regionais e bilaterais. Mais a frente, o texto apresenta a dimensão dos direitos humanos de acesso a medicamentos e a interface entre a medicina tradicional, a propriedade intelectual e o comércio. 
Garantir o acesso a medicamentos essenciais constitui uma obrigação dos Estados no cumprimento dos direitos humanos fundamentais. Nessa conjuntura, os Objetivos de Desenvolvimento do Milênio da $\mathrm{ONU}^{10}$ convocam a todos a desenvolver uma colaboração global que garanta o acesso a medicamentos essenciais. Após a adoção do Acordo sobre os Aspectos dos Direitos de Propriedade Intelectual no âmbito da OMC, a OMS, em colaboração com outras organizações internacionais relevantes, começou a avaliar o impacto dos acordos comerciais que envolviam PI na saúde pública, incluindo a prestação de apoio na implementação das flexibilidades previstas em TRIPS. Outra importante medida é a regulação eficaz, garantindo que os produtos para a saúde sejam providos da qualidade, da segurança e da eficácia necessárias e, assim, assegurando também o fornecimento das informações fundamentais para permitir o uso racional de tais produtos. Se injustificadamente aplicadas, sem critérios claros de proteção à saúde pública, barreiras regulatórias podem dificultar o acesso a tecnologias médicas necessárias e, apesar dos esforços em curso, a harmonização internacional de normas regulamentares continua a ser uma meta distante. Ainda no âmbito da regulação, o surgimento de medicamentos biológicos tem desafiado as autoridades, levantando questões sobre como construir capacidades nacionais para regulamentar produtos biossimilares com base nas diretrizes apropriadas da Organização Mundial da Saúde (WORLD HEALTH ORGANIZATION, 2012).

\subsection{Propriedade Intelectual, Comércio, Saúde e Política}

Segundo o estudo trilateral, as normas multilaterais para cada forma de propriedade intelectual são padrões mínimos, deixando margem para os tomadores de decisões políticas decidirem sobre a sua implementação de uma forma que o sistema de PI seja um suporte para os objetivos de saúde pública. Logo, o sistema de patentes foi projetado para apoiar a inovação e, ao mesmo tempo, oferecer mecanismos para assegurar que tais inovações sejam acessíveis para toda a sociedade. No sistema internacional de propriedade intelectual, um produto, o seu processo de fabricação e sua

\footnotetext{
${ }^{10}$ Os objetivos de desenvolvimento do Milênio da ONU, cujo prazo para cumprimento foi estabelecido para 2015, são compostos por oito metas: erradicação da pobreza e fome extremas; obtenção universal da educação primária; promoção da igualdade de gêneros e a autonomia da mulher; redução da mortalidade infantil; melhoria da saúde materna; combate ao HIV, Malária e outras doenças; assegurar a sustentabilidade ambiental; estabelecimento de parceria global para o desenvolvimento (UNITED NATIONS , 2015a).
}

Revista do Direito Público, Londrina, v.10, n.3, p.51-74, set./dez.2015 | DOI: 10.5433/1980-511X.2015v10n3p74 
utilização podem ser objeto de várias patentes. Portanto, partindo-se de um levantamento eficaz de dados, informações sobre patentes ajudam a determinar a liberdade de operar, determinando em que medida - e com quem - licenças precisam ser negociadas. Ainda no âmbito das patentes, a maneira pela qual os dados de teste são protegidos é relevante para a inovação e para o acesso a medicamentos, e os países têm adotado diferentes regimes de proteção de dados de teste, que vão desde a exclusividade até a publicidade. Avaliando outra modalidade de PI, o sistema de marcas serve para distinguir produtos e informar o consumidor, e, no setor de saúde, as marcas são usadas tanto para medicamentos originais quanto para genéricos. Para evitar confusão, marcas comerciais de produtos farmacêuticos devem ser distintas das Denominações Comuns Internacionais (DCI) dos produtos. Assim, a OMS seleciona a DCI, ou seja, os nomes individuais de aceitação em todo o mundo, para cada substância farmacêutica ativa que está sendo comercializada como um produto farmacêutico, na tentativa de conferir simetria de informação ao processo. Na esfera econômica, a criação de estruturas competitivas de mercado através do direito da concorrência tem um papel importante a desempenhar na melhoria do acesso às tecnologias médicas e na promoção da inovação no setor farmacêutico. Nesse sentido, restrições injustificadas à concorrência, decorrentes do abuso dos direitos de propriedade intelectual (DPI), devem ser tratadas por meio da aplicação de leis pró-concorrência. Afinal, todos os países dependem das importações, em graus variados, para atender às necessidades de saúde de suas populações. Essa dependência de importação é particularmente pronunciada para os sistemas nacionais de saúde dos países de menor desenvolvimento relativo e dos em desenvolvimento. Portanto, processos de compras eficientes, transparentes e competitivos podem contribuir para a melhoria da acessibilidade e da disponibilidade de medicamentos e, consequentemente, para sistemas de saúde mais eficientes e de baixo custo - nesse sentido e para auxiliar os Estados, o Acordo da OMC sobre Contratos Públicos visa promover a transparência e a concorrência leal.

O conhecimento possui as características econômicas do que é comumente chamado de um "bem público", e, por isso, o financiamento do novo conhecimento médico é particularmente difícil. Fatores a serem levados em consideração incluem longos tempos de desenvolvimento de produtos, a necessidade de normas regulamentares rigorosas, o alto risco de fracasso e os 
baixos custos marginais de produção ${ }^{11}$ (especialmente de cópias). Em razão de todos esses fatores, o setor farmacêutico se destaca em termos de sua dependência de patentes para capturar os retornos para pesquisa e desenvolvimento. Todavia, sem desrespeitar as regras internacionais de PI, existem várias opções políticas, dentro e fora do sistema de patentes, para atenuar os altos preços e alavancar os efeitos de bem-estar trazidos por novos produtos, especialmente os farmacêuticos. Os economistas têm apontado que algumas opções podem beneficiar os fabricantes mais do que os consumidores; que a diferenciação de preços poderia desempenhar um papel na redução dos preços nos países mais pobres; e que a falta de proteção da propriedade intelectual e a regulação de preços rigorosa poderiam atrasar o lançamento de medicamentos em certos mercados (WORLD HEALTH ORGANIZATION, 2012).

A medicina tradicional contribui significativamente para a saúde de muitas comunidades e ela é cada vez mais utilizada em países desenvolvidos. Portanto, o reconhecimento apropriado dessa prática de medicina é um elemento importante das políticas nacionais de saúde. Além disso, o crescimento do comércio de produtos de saúde com base no conhecimento tradicional (TK), juntamente com o crescimento do uso de TK como uma vantagem para a investigação biomédica e desenvolvimento de produtos, tem provocado um debate político sobre a apropriação indevida de conhecimento tradicional, além do desenvolvimento - e cumprimento - de protocolos adequados para o acesso e uso de TK. As questões relacionadas com consentimento prévio informado e equitativa repartição de benefícios, garantindo a continuidade de $\mathrm{P} \& \mathrm{D}$, também fazem parte deste debate, e, nesse sentido, o respeito tanto pelo valor econômico quanto pela importância social e cultural do TK é de fundamental importância. Como projeto, a documentação do conhecimento médico tradicional, por meio de bancos de dados e inventários nacionais, pode ser usada como prova do estado da arte em processos de patentes. E, como os países em desenvolvimento utilizam cada vez mais seu TK indígena como base para novos produtos com potencial de exportação significativa, isso cria uma necessidade de regulação da qualidade, segurança e eficácia de tais produtos, o que representa, portanto, mais desafios para os reguladores e produtores (WORLD HEALTH ORGANIZATION, 2012).

\footnotetext{
${ }^{11}$ Custo marginal de produção é "quanto os custos totais aumentam pela produção de uma unidade adicional do produto. Os custos totais aumentam à medida que mais unidades são produzidas. Por que os custos totais são afetados pela lei dos rendimentos decrescentes, o custo de cada unidade adicional da produção aumenta (custo marginal crescente)" (NÚCLEO DE ESTUDOS DE ECONOMIA DO MEIO AMBIENTE, 2015).
} 


\subsection{Inovação}

O estudo desenvolvido pelas Agências da ONU fornece uma visão detalhada sobre questões políticas relativas à dimensão inovação de tecnologias médicas. O padrão histórico da pesquisa médica e desenvolvimento provê um pano de fundo para a análise de tendências atuais no cenário $P \& D$. $O$ texto aborda ainda o desafio da inovação representado por doenças negligenciadas, além de instrumentos alternativos e complementares de apoio à pesquisa e desenvolvimento, descrevendo o papel dos DPI no ciclo de inovação. Depois de descrever os principais elementos do cenário da política de inovação e acesso, o estudo trilateral analisou a forma como o atual enquadramento se aplica à inovação nessas tecnologias, mensurando os fatores que estimularam a inovação em tecnologias médicas no passado, identificando como os modelos atuais de P\&D estão evoluindo e traçando o papel de participantes no processo de inovação, incluindo-se o contexto das doenças negligenciadas. O trabalho das três Agências também abordou questões levantadas na área de propriedade intelectual, em particular o sistema de patentes, refletindo o fato de que as políticas de saúde na última década terem dado maior atenção à dimensão da inovação e considerando, em particular: o tipo de estruturas de colaboração; mecanismos de incentivo; fontes de financiamento; ferramentas necessárias para a construção de processos de inovação mais amplos; e, ainda, como garantir que as atividades de pesquisa médica se concentrem cada vez mais em áreas historicamente negligenciadas.

O processo de pesquisa e desenvolvimento no setor farmacêutico moderno evoluiu em empresas de grande porte, geralmente de propriedade privada, onde, tradicionalmente, $\mathrm{P} \& \mathrm{D}$ e marketing eram realizados internamente. No começo, a produção foi amplamente licenciada por empresas de medicamentos de referência e, mais tarde, porém, a comercialização e a distribuição de novos produtos eram geralmente realizadas através de um sistema de direitos exclusivos a fornecedores únicos. Atualmente, o modelo convencional de inovação em tecnologias médicas enfrenta desafios consideráveis, incluindo a evolução dos mercados, os custos mais elevados, e as normas regulamentares mais rigorosas. No âmbito político, o setor público impacta significativamente o ciclo de inovação em várias etapas, fornecendo diretamente entradas de P\&D, ajudando a moldar as prioridades de pesquisa e desenvolvimento das empresas privadas e influenciando como os produtos para a saúde são adquiridos e disseminados. Ainda na avaliação da influência política, criar produtos farmacêuticos e trazêlos para o mercado é geralmente caro e demorado. No entanto, os dados limitados 
dificultam uma avaliação confiável, independente dos verdadeiros custos de pesquisa médica. Existem muitos mecanismos para a promoção da inovação, e os direitos de propriedade intelectual formam uma estrutura de incentivos útil, mas é bastante discutível se o sistema de PI pode incentivar invenções em áreas onde não há mercado - mais um motivo para uma intervenção estatal nas situações nas quais se identifique tais falhas. Nesse diapasão, a Comissão da OMS sobre Direitos de Propriedade Intelectual, Inovação e Saúde Pública constatou que o ciclo de inovação é autossustentável em países industrializados, que têm um grande mercado, permitindo assim que as empresas recuperem seus investimentos, mas este não é o caso nos países de baixa renda, onde os mercados são pequenos e os serviços de saúde carecem de recursos. Portanto, instrumentos de política de inovação podem diferir à proporção que se dirigem a pesquisa publicamente financiada e/ou executada. As vacinas são um problema pontual, pois, diferentemente da maioria dos medicamentos, muito raramente há opções de vacinas genéricas, já que o processo de comprovação de segurança e eficácia desses imunizadores, mesmo que se trate de uma cópia, requer sempre um marco regulatório completo. Ainda assim, nos últimos anos, tem ocorrido um aumento significativo no desenvolvimento de novas vacinas e de novos modelos de inovação, juntamente com um número crescente de fabricantes de vacinas em LMICs. Para facilitar o processo, dando publicidade ao sistema, a OMS estabeleceu um registro de ensaios clínicos que faz com que dados estejam publicamente disponíveis, no cumprimento dos interesses da saúde pública e da ciência (WORLD HEALTH ORGANIZATION, 2012).

De acordo com o estudo trilateral, a inovação em tecnologias médicas para doenças negligenciadas sofre de falhas de mercado adicionais e, assim, os incentivos baseados em PI não são suficientes para gerar investimento adequado em pesquisa e desenvolvimento. Mesmo havendo uma lacuna, o cenário de P\&D das doenças negligenciadas está mudando, e um número crescente de atores vem se envolvendo no financiamento e na execução de tais pesquisas. Muitos dos novos mecanismos de inovação e modelos que visam aumentar a P\&D para encontrar tratamentos eficazes para doenças negligenciadas estão sendo discutidos e implementados em nível nacional e internacional. As avaliações de algumas dessas propostas podem ser encontradas nos relatórios publicados pelo Grupo de Trabalho de Especialistas da OMS para a Investigação e Desenvolvimento: Financiamento e Coordenação e pelo Grupo de Trabalho de Peritos Consultivo para a Investigação e Desenvolvimento: Financiamento e 
Coordenação $\left(\mathrm{CEWG}^{12}\right)$. Os relatórios recomendam pontos de ação específicos, incluindo a criação de um instrumento global vinculativo para $\mathrm{P} \& \mathrm{D}$ para o tratamento dessas doenças em países em desenvolvimento. Noutra frente de batalha, as Parcerias de Desenvolvimento Produtivo ( $\mathrm{PDP}^{13}$ ) têm aumentado significativamente o número de produtos em desenvolvimento para as doenças que afetam predominantemente países de baixa e média rendas.

O quadro jurídico internacional que rege os DPI e, mais importante, as escolhas feitas nesse âmbito, nacional e regionalmente, pode ser uma determinante essencial para o ciclo de inovação. O papel das leis que regulam as patentes no desenvolvimento de novas tecnologias médicas depende não só do design legal e administrativo do sistema, mas também de decisões específicas feitas por partes individuais. Isso ocorre em diferentes fases do processo de desenvolvimento, em termos de saber se, e quando, obter direitos de patente e como exercê-los. Nessa área de discussão, os avanços da biotecnologia ${ }^{14}$ no campo da inovação médica levaram a um renovado debate sobre o que deve ser considerado ou não matéria patenteável e como identificar a aplicabilidade industrial em tais casos. Outro ponto de discussão diz respeito à inovação incremental, que pode melhorar a segurança, o efeito terapêutico ou do método de administração de um medicamento ou vacina existente. Nacionalmente, se tais invenções merecem a concessão de uma patente, trata-se de uma análise caso a caso, e, embora a patente de inovação incremental não estenda o prazo das originais, existem preocupações sobre os efeitos negativos de tais estratégias de patenteamento. Essas estratégias, além de tantas outras, são chamadas de "perenização" (evergreening) e continuam sendo uma questão de bastante

${ }^{12}$ O Grupo de Trabalho de Peritos Consultivo para a Investigação e Desenvolvimento: Financiamento e Coordenação foi criado como resposta a uma preocupação de que os recursos destinados à pesquisa e desenvolvimento relacionados ao tratamento de doenças que afetam sobretudo os países em desenvolvimento eram insuficientes. Tal grupo sucedeu o antigo Grupo de Trabalho de Especialistas da OMS para a Investigação e Desenvolvimento: Financiamento e Coordenação, e sua função atual é, dentre outras, continuar e aprofundar as análises de seu predecessor (WORLD HEALTH ORGANIZATION, 2012).

13 "O Ministério da Saúde tem buscado investir cada vez mais em parcerias para a produção de medicamentos e vacinas. Atualmente, o Ministério possui 104 parcerias de desenvolvimento de produtos (PDP) envolvendo 19 laboratórios públicos e 57 privados. Esses acordos preveem o desenvolvimento de 101 produtos ( 66 medicamentos, 7 vacinas e 28 produtos para saúde).As PDP são destinadas à transferência de tecnologia entre instituições públicas e privadas e às encomendas tecnológicas vinculadas às demandas de produtos estratégicos para SUS. Esses acordos devem gerar uma economia de R\$ 4,1 bilhões por ano em compras públicas" (BRASIL, 2014).

${ }^{14} \mathrm{De}$ acordo com a convenção sobre diversidade biológica, biotecnologia é qualquer aplicação tecnológica que utiliza sistemas biológicos, organismos vivos ou seus derivados, com a finalidade de produzir ou modificar produtos ou processos para utilização específica (UNITED NATIONS, 2015b). 
controvérsia. Algumas leis de patentes permitem, ainda, a concessão de proteção sobre um produto para o qual foi identificada uma nova indicação médica, mas apenas na condição de que o produto proposto compra todos os critérios de patenteabilidade, mas essa modalidade também tem sido fonte de debate internacional. Na esfera das exceções à proteção patentária, enquanto a pesquisa é uma das exceções mais comumente encontradas nas leis nacionais de PI, não há uma abordagem única utilizada mundialente, e nem todos os países fazem uso de tais exceções. As licenças, por sua vez, são ferramentas para a construção de parcerias e cooperações e podem permitir a entidades do setor público o alcance dos objetivos de políticas públicas. Um exemplo ocorreu durante a preparação para uma pandemia de gripe da OMS, quando se realizou o compartilhamento de vírus da gripe e o acesso a vacinas. A preparação também permitiu a repartição de benefícios derivados desses vírus, incluindo a gestão da propriedade intelectual. Os acordos de transferência de materiais padrão naquela ocasião estipularam que os laboratórios participantes não deviam obter direitos de propriedade intelectual em material biológico. Além disso, esses acordos previam um leque de opções para os destinatários de materiais biológicos, como os fabricantes de vacinas contra a gripe, a celebrar acordos de repartição de benefícios (WORLD HEALTH ORGANIZATION, 2012).

\subsection{Acesso}

O estudo trilateral lida, também, com os aspectos fundamentais da dimensão de acesso, descrevendo o contexto atual para o acesso a tecnologias médicas essenciais, e definindo os principais determinantes de acesso relacionados com os sistemas de saúde, PI e comércio internacional. Nesse sentido, é preciso realmente uma análise, em especial, das políticas de preços, taxas e sobretaxas, dos mecanismos de aquisição, bem como dos aspectos e iniciativas regulatórias para transferir tecnologia e aumentar a produção local, a qualidade das patentes e procedimentos de revisão, licenças compulsórias e voluntárias, acordos comerciais, tarifas e política de concorrência. $\mathrm{O}$ acesso a medicamentos e outras tecnologias médicas é parte de um desafio mais amplo de garantir a promoção aos cuidados de saúde, o que requer um sistema de saúde nacional em funcionamento. A melhoria do acesso aos medicamentos e demais tecnologias só irá proporcionar benefícios para a saúde pública se os produtos ofertados forem produtos de qualidade. O preço é um fator determinante do acesso aos medicamentos, especialmente nos países nos quais o setor público de saúde é incipiente e onde o tratamento é comprado no mercado privado. 
Ainda com relação ao preço, em geral, os medicamentos genéricos são mais baratos do que os produtos originais, mas, mesmo os genéricos de baixo preço, muitas vezes, ainda são inacessíveis para grande parte da população em países de renda baixa e média. Desde 2001, um aumento significativo do financiamento internacional para medicamentos essenciais (especialmente para HIV, malária e tuberculose, além de vacinas) melhorou o acesso a esses produtos em países periféricos.

Um pré-requisito fundamental para um sistema de saúde em funcionamento é o compromisso do governo de forma adequada e sustentável financiar o sistema nacional de saúde e o fornecimento confiável de medicamentos essenciais. A maior disponibilidade de antirretrovirais (ARV) de qualidade, a preços acessíveis, tem sido responsável por um aumento dramático no número de pacientes com HIV em tratamento. Todavia, enquanto muitos dos tratamentos mais antigos estão disponíveis a partir de fontes genéricas, os ARV mais recentes ainda estão protegidos por patentes na maioria dos países. A Declaração Política das Nações Unidas sobre HIV obriga os Estadosmembros da ONU a remover os obstáculos que limitem a capacidade dos países periféricos para fornecer meios eficazes de prevenção e tratamento. Entre essas medidas, incluem-se flexibilidades do TRIPS, a promoção da concorrência por meio de genéricos, o compartilhamento aberto de direitos de propriedade industrial e os pools de patentes. Em muitos países periféricos, a disponibilidade de medicamentos para enfermidades crônicas continua inferior à dos medicamentos para doenças agudas, embora tais tratamentos essenciais estejam disponíveis a preços baixos (WORLD HEALTH ORGANIZATION, 2012).

Segundo as três Agências da ONU, países desenvolvidos usam uma variedade de medidas para aumentar a quota de mercado dos medicamentos genéricos a preços acessíveis, a fim de controlar os orçamentos de saúde. Seguindo o modelo, os LMICs poderiam gerar poupanças adicionais através da implementação desse tipo de medida. Enquanto a diferenciação de preços pode ser usada como uma ferramenta complementar para aumentar o acesso, o compromisso do governo de proporcionar o acesso a medicamentos para aqueles que não podem pagar por eles continua a ser essencial. Além disso, os sistemas de aquisição devem ser projetados para obter medicamentos selecionados e outros produtos médicos de boa qualidade, no tempo certo, nas quantidades necessárias e com custos favoráveis, e a aquisição conjunta pode contribuir para a redução de custos no processo de compra. As informações sobre patentes sobre produtos específicos em mercados específicos pode facilitar a aquisição 
de medicamentos genéricos, já que as tendências mostram que a produção local está crescendo e se diversificando em alguns países periféricos através de esforços nacionais e inúmeras iniciativas regionais e internacionais.

A mera existência de direitos de propriedade intelectual sobre um produto não é uma barreira para o acesso a esse produto, e o impacto dos DPI sobre o acesso a tecnologias médicas depende de como eles são nacionalmente regulados e de como eles são gerenciados pelo titular do direito. Os membros da OMC têm flexibilidade para projetar seus sistemas nacionais de propriedade intelectual dentro dos padrões mínimos estabelecidos em TRIPS, incluindo seus objetivos econômicos, de desenvolvimento e de saúde pública. A definição de critérios de patenteabilidade e sua aplicação na prática podem ter um impacto considerável sobre o acesso a tecnologias médicas, além de implicações para a entrada no mercado de produtores de genéricos. Os membros da OMC são livres, também, para determinar as bases para a concessão de licenças compulsórias e estas razões podem incluir o interesse público em geral e não se limitam a emergências de saúde pública. Eventualmente, as licenças compulsórias e autorizações de uso do governo podem ser usadas para importar medicamentos genéricos mais baratos ou para produzi-los localmente. Nos termos de TRIPS, os membros da OMC são livres para determinar seu regime exaustão, que é um regime de esgotamento internacional, permitindo a importação paralela de medicamentos patenteados. Alguns países permitem o prazo da patente a ser prorrogado, a pedido do titular da patente ou não, para compensar os atrasos regulatórios e outros, e diferentes pontos de vista foram expressos sobre o impacto de tais extensões na saúde pública. As empresas usam cada vez mais licenças voluntárias como parte de seus programas de responsabilidade social, especialmente na área de HIV, e essa tendência foi reforçada pela criação do Pool de Patentes de Medicamentos (MPP $\left.{ }^{15}\right)$. As disposições mais comuns em acordos de livre comércio que afetam o setor farmacêutico são definição de critérios de patenteabilidade, extensões de prazo de patentes, proteção de dados de teste, ligação de aprovação regulatória com patentes e aplicação de DPI, incluindo as medidas de fronteira. Os Estados precisam estar atentos, pois essas disposições podem atrasar a entrada dos genéricos no mercado e aumentar os preços dos medicamentos (WORLD HEALTH ORGANIZATION, 2012).

\footnotetext{
${ }^{15}$ O Medicines Patent Pool é uma organização fundada em 2010 cujo objetivo é a redução de preços dos medicamentos de HIV e também a facilitação do desenvolvimento de adaptações melhoradas desses fármacos, além de especiais formulações para crianças, por meio de licenças voluntárias e de pool de patentes (MEDICINES PATENT POOL, 2015).
} 
Ainda de acordo com o estudo trilateral, o comércio internacional é fundamental para garantir o acesso a medicamentos e outros produtos médicos. Os países em desenvolvimento, os LDCs e as economias em transição compreendem $85 \%$ da população mundial, mas são responsáveis por apenas $30 \%$ das importações e de $20 \%$ das exportações de produtos comercializados internacionalmente relacionadas com a saúde. A política de concorrência é relevante para todas as fases do processo de fornecimento de tecnologia médica para os pacientes - desde o desenvolvimento e fabricação de tecnologia médica até a sua eventual venda e entrega. Portanto, a política de concorrência tem um papel importante a desempenhar na prevenção de conluio entre os fornecedores de tecnologia médica que participam nos processos de aquisições.

\section{CONSIDERAÇÕES FINAIS}

Em evento realizado no Instituto Nacional de Propriedade Industrial $\left(\mathrm{INPI}^{16}\right)$, a conselheira da divisão de propriedade intelectual da OMC, Sra. Jayashree Watal, traçou um amplo painel da evolução dos tratados internacionais relacionados com saúde, propriedade intelectual e comércio. Para a Sra. Watal, o grande desafio nas discussões é como conciliar a necessidade de garantir investimentos em pesquisa, por meio de patentes, e a de permitir que os países menos desenvolvidos possam viabilizar uma indústria de medicamentos e, principalmente, permitir que populações mais pobres tenham acesso a produtos médicos com preços diferenciados (INSTITUTO NACIONAL DA PROPRIEDADE INDUSTRIAL, 2013).

O Brasil, por sua vez, além de permitir a inclusão do contestável instituto do pipeline, artigo 230 da LPI (Lei de Propriedade Industrial - Lei No 9.279, de 14 de maio de 1996), não se aproveitou da prerrogativa de prazo estabelecida pelo TRIPS, validando os direitos de propriedade intelectual estabelecidos pelo Acordo pouco após a ratificação do tratado. À época, essa falta de análise de prazos, somada à figura controvertida do pipeline, foi a demonstração da uma clara falta de estratégia do governo brasileiro no estabelecimento de políticas públicas de incentivo à PI e de acesso à saúde pública.

\footnotetext{
${ }^{16}$ Criado em 1970, o Instituto Nacional da Propriedade Industrial (INPI) é uma autarquia federal vinculada ao Ministério do Desenvolvimento, Indústria e Comércio Exterior (MDIC), responsável pelo aperfeiçoamento, disseminação e gestão do sistema brasileiro de concessão e garantia de direitos de propriedade intelectual para a indústria. Entre os serviços do INPI, estão os registros de marcas, desenhos industriais, indicações geográficas, programas de computador e topografias de circuitos, as concessões de patentes e as averbações de contratos de franquia e das distintas modalidades de transferência de tecnologia (INSTITUTO NACIONAL DA PROPRIEDADE INDUSTRIAL, 2015).
} 
O estudo elaborado pelas três Agências da ONU fornece uma plataforma sólida para o debate político e para a formulação de outras análises sobre o tema, sendo, portanto, uma forte ferramenta de avaliação de cenários, nacionais e internacionais, para os tomadores de decisões políticas, visando o acesso à saúde pública, sem, contudo, frear os incentivos às inovações em tecnologias médicas. Nesse sentido, o Brasil, sendo membro ativo da OMS, no intuito de evitar os erros causados pela falta de análise de cenários do passado, deve se apropriar das informações contidas no estudo trilateral para, doravante, tomar decisões políticas mais acertadas.

\section{REFERENCIAS}

BRASIL. Ministério da Saúde. Ministro da Saúde apresenta Parcerias de Desenvolvimento Produtivo para empresários. 2014. Disponível em: $<$ http://www.brasil.gov.br/saude/2014/09/ ministro-da-saude-apresentaparcerias-de-desenvolvimento-produtivo-para-empresarios $>$. Acesso em: 10 ago. 2015.

BRASIL. Ministério da Saúde. Secretaria de Ciência, Tecnologia e Insumos Estratégicos (SCTIE). 2013. Disponível em: $<$ http:// portalsaude.saude.gov.br/index.php/o-ministerio/principal/secretarias/sctie > Acesso em: 10 ago. 2015.

GADELHA, Carlos Augusto Grabois; COSTA, Laís Silveira; MALDONADO, José. O Complexo Econômico-Industrial da Saúde e a dimensão social e econômica do desenvolvimento. Revista de Saúde Pública, 2012. Disponível em: $<$ http://www.scielo.br/ scielo.php?script $=$ sci_arttext\&pid=S0034-89102012000700004 $>$. Acesso em: 10 ago. 2015.

INSTITUTO DE ESTUDOS DO COMÉRCIO E NEGOCIAÇÕES INTERNACIONAIS. Glossário. 2015. Disponível em: $<$ http://www.icone brasil.com.br/biblioteca/glossario/letra/p>. Acesso em: 10 ago. 2015.

INSTITUTO NACIONAL DA PROPRIEDADE INDUSTRIAL. Especialista da OMC analisa acordos relacionados à PI e saúde. 2013. Disponível em: $<$ http://www.inpi.gov.br/portal/artigo/especialista_da_omc_ analisa_acordos_relacionados_a_pi_e_saude>.Acesso em: 18 jun. 2013. 
INSTITUTO NACIONAL DA PROPRIEDADE INDUSTRIAL. O INPI. 2015. Disponível em: $<$ http://www.inpi.gov.br/sobre/estrutura $>$. Acesso em: 10 ago. 2015.

MEDICINES PATENT POOL. About de MPP. 2015. Disponível em: $<$ http:/ /www.medicinespatentpool.org/about/>. Acesso em: 10 ago. 2015.

NÚCLEO DE ESTUDOS DE ECONOMIA DO MEIO AMBIENTE. Mercados, Demanda, Oferta, e Bem-estar Social. 2015. Disponível em: $<$ http://www.neema.ufc.br/Ern_cap2_parte2.htm>. Acesso em: 10 ago. 2015.

OCDE - Organização para a Cooperação e Desenvolvimento Econômico. Manual de Oslo - Proposta de Diretrizes para Coleta e Interpretação de Dados sobre Inovação. São Paulo, 2005.

UNITED NATIONS. The Convention. 2015. Disponível em: $<$ https:// www.cbd.int/ convention/articles/default.shtml? $\mathrm{a}=\mathrm{cbd}-02>$. Acesso em: 10 ago. 2015.

UNITED NATIONS. Millennium Development Goals and beyond 2015. 2015a. Disponível em: $<$ http://www.un.org/millenniumgoals/>. Acesso em: 12 jul. 2015.

UNITED NATIONS. Overview. 2015b. Disponível em: $<$ http://www.un.org/ en/ sections/about-un/overview/index.html>. Acesso em: 6 ago. 2015.

WORLD HEALTH ORGANIZATION. Consultative Expert Working Group on Research and Development: Financing and Coordination. 2012. Disponível em: $<$ http://www.who.int/phi/cewg/en/>. Acesso em: 10 ago. 2015.

WORLD HEALTH ORGANIZATION. Global Strategy and Plan of Action on Public Health, Innovation and Intellectual Property. 2011. Disponível em: $<$ http://www.who.int/phi/publications/Global_Strategy_ Plan_Action.pdf?ua=1>. Acesso em: 10 ago. 2015. 
WORLD HEAlth ORGANIZATION. Promoting Access to Medical Technologies and Innovation - Intersections between public health, intelectual property and trade. Geneva: WHO \& WIPO \& WTO, 2012.

WORLD HEALTH ORGANIZATION. The Global Strategy and Plan of Action on Public Health, Innovation and Intellectual Property. 2015. Disponível em: $<$ http://www.who.int/phi/implementation/phi_globstat_action/ en/>. Acesso em: 10 ago. 2015.

WORLD HEALTH ORGANIZATION. World Health Organization. Disponível em: $<$ http://www.who.int/en/>. Acesso em: 29 jul. 2013.

WORLD INTELLECTUAL PROPERTY ORGANIZATION. Development Agenda for WIPO. 2015. Disponível em: $<$ http:// www.wipo.int/ip-development/en/agenda/>. Acesso em: 10 ago. 2015.

WORLD INTELLECTUAL PROPERTY ORGANIZATION. World Intellectual Property Organization. 2013. Disponível em: $<$ http:// www.wipo.int/portal/index.html.en>. Acesso em: 20 maio 2013.

WORLD TRADE ORGANIZATION. The Doha Declaration explained. 2015. Disponível em: $<$ https://www.wto.org/english/tratop_e/dda_e/ dohaexplained_e.htm>. Acesso em: 10 ago. 2015.

WORLD TRADE ORGANIZATION. World Trade Organization.

Disponivel em: <http://www.wto.org/>. Acesso em: 29 jul. 2013.

Artigo recebido em: 11/09/2015 Aprovado para publicação em: 26/10/2015

Como citar: MENDES, Dany Rafael Fonseca. NOGUEIRA, Tatiana Siqueira. OLIVEIRA, Michel Angelo Constatino de. O Estudo Trilateral (OMPI, OMC, OMS) e a Promoção do Acesso à Saúde. Revista do Direito Público. Londrina, v.10, n.3, p.51-74, set./dez.2015. DOI: 10.5433/1980511X.2015v10n3p51. ISSN: 1980-511X. 